\title{
LA MUJER JOVEN EN LOS CARGOS DE DIRECCIÓN Y LAS RELACIONES DE GÉNERO EN EL SECTOR AGROPECUARIO DE LA PROVINCIA CIENFUEGOS. ESTUDIOS DE CASOS
}

\section{YOUNG WOMEN IN MANAGEMENT POSITIONS AND GENDER RELATIONS IN THE AGRICULTURAL SECTOR OF CIENFUEGOS PROVINCE. CASE STUDIES}

${ }^{1}$ Universidad de Cienfuegos, CINTIA BEATRIZ PÉREZ LANZA ${ }^{1}$

\begin{abstract}
Resumen
Los estudios históricos desde la mirada de género han demostrado que, no obstante de los avances de la mujer en la vida pública, todavía se encuentra presente la diferencia de oportunidades en el acceso a cargos de dirección, a pesar de existir la voluntad política de los estados del derecho a la igualdad de sexo en el acceso al empleo y a los cargos de responsabilidad, sin embargo, en el Sector de la Agricultura en la provincia de Cienfuegos la presencia de la mujer en cargos de dirección es insuficiente. El objetivo de la presente investigación consiste en valorar la situación de la mujer en
\end{abstract}


los cargos de dirección y las relaciones de género que se manifiestan en el sector agropecuario de la provincia Cienfuegos, a través del paradigma mixto con 5 estudios de casos que muestran la diferencia de género en los cargos principales de dirección. Esto requiere de reflexión, análisis y de una estrategia, para elevar la integración de la mujer a la función directiva, a fin de favorecer el desarrollo social, la realización de los derechos humanos, el mejoramiento de la calidad de vida y el desarrollo territorial.

Palabras claves: dirección, empoderamiento, acceso, empleo, desarrollo

\section{Abstract}

The historical studies from the gender perspective have demonstrated that in spite of the women advances in the public life, the differences in opportunities is still present in the access to management positions, in spite the political will of the states from the right to sex equality in the access to employment and the positions of responsibility, in the Agriculture sector in Cienfuegos province, the woman's presence in this positions are insufficient. The objective of this investigation consists on valuing the women situation in the management positions and the gender relationships that are manifested in Cienfuegos' agricultural sector, through the mixed paradigm with 5 case studies that show the gender 
difference in the main positions of management, which requires the reflection, analysis and strategy to elevate the integration of the women to the executive duty, in order to favor the social development, the realization of the human rights, the improvement of the quality of life and the territorial development.

Keywords: address, empowerment, access, employment, development

RECEPCIÓN: 11 DE JULIO DE 2018/ACEPTACIÓN: 8 DE OCTUBRE DE 2018

\section{EL GÉNERO COMO CATEGORÍA DE}

ANÁLISIS

La comprensión del abordaje del término género requiere de un amplio conocimiento sobre sus antecedentes históricos y fundamentación teórica, lo que facilita la realización de investigaciones con este enfoque, que posibiliten obtener resultados sobre las diferencias entre mujeres y hombres, y poder intervenir de forma equitativa sobre las desventajas de cada uno.

El concepto de género proviene de países anglosajones, es una traducción que se entiende en inglés como referente a relaciones entre los sexos. En las ciencias sociales y en la vida cotidiana se utiliza para referirse al condicionamiento sociohistórico-cultural que asigna a hombres y a mujeres determinadas funciones. En tanto, en este último sentido, a los efectos 
del desarrollo de la presente investigación, al hablar del término género, se refiere a la relación entre personas de diferentes sexos, de diferencias aprendidas desde la infancia a través de los padres y madres, de las familias, de los amigos y vecinos, reforzadas a todo lo largo de la vida por los grupos humanos en los cuales se participa y por los medios de comunicación social, por lo tanto, género no es lo mismo que sexo, el sexo (hembra o varón, femenino o masculino) describe las diferencias biológicas entre hombres y mujeres, diferencias que son morfológicas, propias de nuestra naturaleza y generalmente no son cambiables, en cambio, el género es una construcción social de las diferencias sexuales en un momento o lugar histórico dado (Fuller, 2006, p. 7).

El vocablo género como categoría crucial para la teoría feminista tiene sus orígenes en la obra de John Money, quien en 1951 usa el concepto gender (género) por primera vez para referirse al componente cultural, fundamentalmente la influencia educativa, en la formación de la identidad sexual. La identidad sexual era vista sólo como determinada biológicamente, por lo que el uso de este concepto para referirse a un aspecto que la cultura forma, constituye un aporte importante al conocimiento científico (Vasallo, 2004, p. 21). Con posterioridad Robert Stoller, en su libro Sex and Gender (Sexo y Género) utiliza formalmente la expresión "identidad genérica" y a partir del estudio de los trastornos de la identidad sexual, estable- 
ció la diferencia entre sexo y género (Stoller, 1968; citado en Fuller, 2006, p. 7).

Los valiosos aportes de Money y Stoller en la conceptualización sobre el género, han contribuido en la elaboración de nociones complejas, que dieran cuenta de sus múltiples dimensiones. Mientras que el sexo hace referencia a los componentes biológicos que determinan que una persona sea varón o hembra, el término género hace referencia a aquellos componentes psicológicos y culturales que forman parte de las definiciones sociales de las categorías hombre y mujer, distinción con la cual concuerdan los teóricos que trabajan en este campo (Fuller, 2006, p. 18).

Otro aporte a la interacción de la persona con su contexto social para la conformación del género, que abrió un nuevo campo de indagación intelectual sobre la interpretación de la igualdad y la diferencia de los sexos, fueron las ideas de Simone de Beauvoir, al plantear que cada persona no nace, es construida, y así lo señaló de manera brillantísima en el libro El Segundo Sexo: "no nacemos mujeres, nos hacemos mujeres" (De Beauvoir, 1981; citado en Baute, 2010, p. 27).

Existe una amplia bibliografía que aborda dos términos distintos para referirse a las diferencias biológicas y aquellas construidas socialmente, éstos son sexo y género respectivamente, y aun cuando ambos se relacionan con las diferencias entre las mujeres y los hombres, las nociones de género y sexo tienen connotaciones distintas. 
Para Scott (Scott, 1996, p. 89), género es una forma primaria de las relaciones significantes de poder y es utilizada como: categoría de análisis; construcción social; poder, porque determina quién ejerce el poder y sobre quién; relaciones: hombre-mujer, mujeres-mujeres, hombres-hombres, más otras relaciones; ecología, raza, etnia. Introduce la dimensión histórica y del poder relacional en estas diferencias. Alerta sobre la expresión del poder en la construcción cultural de las desigualdades a partir de las diferencias de género, al respecto nos advierte que

el género es un elemento constitutivo de las relaciones sociales basado en las diferencias percibidas entre los sexos, y el género es una primera forma de significar las relaciones de poder. Es el primer campo en el cual el poder es articulado. (Scott, 1996; citado en Fuller, 2006, p. 13)

Para estudiar la categoría de género se debe tener en cuenta que la misma puede definirse como: conjunto de características culturalmente específicas que identifican el comportamiento social de hombres y mujeres y la relación entre ellos, basada en la diferenciación de sexo, por lo que se asume el concepto de género como el proceso social condicionante que establece rasgos diferenciadores y conductas estereotipadas (Nuñez, 2001, p. 35). 


\section{ESTUDIOS ACERCA DEL PAPEL DE LA}

\section{MUJER DIRIGENTE}

Los estudios sobre mujeres ejecutivas han ganado legitimidad en la medida en que aumenta la participación de las mujeres profesionales en el mercado laboral y asumen en forma creciente roles importantes en la dirección y los niveles medios de las empresas públicas y privadas. Estudios sobre mujeres en puestos de dirección en España llevados a cabo por investigadoras del Instituto de la Mujer, muestran que las mujeres tienen un estilo de dirección propio, que difiere en muchos casos del estilo de dirección fuerte y duro que se adjudica a la dirección de los hombres, y sin los cuales es imposible ejercer el poder. Esto indica que, más allá de los estereotipos, la presencia femenina en actividades de poder y prestigio, significa un cambio importante en la manera en que se conciben hoy estos espacios de poder.

A pesar de estos resultados, algunos investigadores se cuestionan la existencia de cambios con la presencia femenina en la dirección. En este sentido, los investigadores europeos, Smircich y Calas (1983), en atención a las especificidades de sus países, han desarrollado estudios para conocer si son reales los avances de las mujeres en puestos directivos o si este hecho no constituye una repetición del ya tradicional reparto de tareas en el hogar y fuera de éste, sólo que en este caso a un nivel superior y de mayor complejidad. Mientras Snavely (1993) 
duda de la capacidad de las mujeres para influir al mismo nivel de sus compañeros masculinos.

Mientras tanto los investigadores europeos, Kesner (1989) y Jacobs (1992) al analizar el contenido del trabajo directivo de las mujeres llegan a dos importantes conclusiones: que en muchas empresas cabe hablar de una fachada, para justificar con el acceso de las mujeres al poder la igualdad de oportunidades, y que través de un exceso de paternalismo las mujeres estén en la dirección sólo como figuras decorativas (Jacobs, 1992, p. 33)

En América Latina existen investigaciones actuales sobre el tema, cuyos resultados arrojan una información muy valiosa para este estudio. De los trabajos pioneros se reseña los de las investigadoras del Colegio de México María Luisa Tarrés (1992), Julia Tuñón y Soledad González (1997) que han desarrollado estudios acerca del perfil de la mujer empresaria en México. Estas autoras refuerzan a partir de los resultados de sus investigaciones la teoría planteada anteriormente acerca del efecto transformador que ejercen las mujeres en el sistema de valores masculinos, pues éstas al alcanzar el éxito económico encuentran reacomodo en un mundo al que sienten que no pertenecen completamente pero donde a largo plazo se insertarán y lograrán un equilibrio entre las responsabilidades familiares y las exigencias laborales (Tarrés, 1992, p. 12).

Estos resultados apuntan en otra dirección, aunque no dejan de ser tenidos en cuenta a partir de los esquemas de domina- 
ción de las mujeres en las familias y la reproducción de estos esquemas en la dirección de las empresas. Como resultado de estos estudios puede incorporarse las reflexiones un conjunto de elementos, con independencia de que la investigación se realice en el contexto cubano. Uno de los objetivos principales de estos estudios ha sido medir y caracterizar la participación femenina según sectores y niveles directivos, mientras otros han explorado las dinámicas organizacionales, los menos son los que se refieren al desarrollo de las carreras profesionales y los estudios de la identidad (González, 2005, p. 67).

\section{ESTUDIOS ACERCA DEL PAPEL DE LA MUJER DIRIGENTE Y SU PARTICIPACIÓN EN LA ACTIVIDAD ECONÓMICA PRODUCTIVA DE LA SOCIEDAD CUBANA}

En Cuba los estudios sobre el acceso de las mujeres a la toma de decisiones son recientes y se han desarrollado en las diferentes áreas de la investigación social atendiendo a la presencia femenina en actividades particulares de dirección como la económica, la social y la política.

Según la investigadora Mayda Álvarez, estas investigaciones pueden ser clasificadas en dos grupos: a) las que indagan acerca del acceso de la mujer a la toma de decisiones en las diferentes esferas de la vida económica, política y social, y los obstáculos para este acceso, y b) las que tratan particular- 
mente acerca de la representación femenina en los Órganos del Poder Popular (Álvarez, 1999, p. 10).

Otro grupo de estudios, (Díaz y Calderón, 1997, p. 4) al correlacionar tres ejes fundamentales: dirección, trabajo y familia, a través del planteo de interrogantes relacionadas con las dificultades para lograr el acceso y la conciliación de los espacios laborales, de dirección y doméstico, han brindado resultados que demuestran la existencia de factores objetivos y subjetivos que impiden el acceso de las mujeres a la dirección en la esfera laboral. Los resultados demuestran que existen barreras sociales relacionadas con elementos psicológicos y culturales que limitan la dirección femenina y que son elementos muy sutiles, pero que son considerados como signos de discriminación hacia las mujeres.

De acuerdo con los estudios de la investigadora cubana Graciela González Olmedo (González, 2005, p. 45) existen dos importantes vertientes de análisis que coinciden en la necesidad cada vez más apremiante de lograr la plena asimilación de la mujer en el desempeño de la labor directiva.

REALIDADES Y RETOS DE LOS ESTUDIOS DE GÉNERO EN SECTORES RURALES Y AGROPRODUCTIVOS CUBANOS

Las elaboraciones históricas de los géneros se basan en sistemas de poder, con un discurso hegemó- 
nico que genera la existencia de los conflictos sociales. La problematización de las relaciones de género logró romper con la idea del carácter natural de las mismas. Desde el triunfo de la Revolución en 1959, el estado del sector agropecuario cubano se desarrolla en un escenario con particulares marcas patriarcales en las relaciones de género, no obstante, se aprecian avances relevantes en materia de igualdad. Entre los resultados pueden mencionarse los siguientes adelantos alcanzados por las mujeres (Hernández y Arteaga, 2013, p. 54):

- El derecho al trabajo se hace realidad en las áreas rurales cubanas a partir de que cada día las mujeres se van incorporando a sectores como el agropecuario, que en épocas pasadas eran preferentemente de hombres.

- Las mujeres han recibido las tierras como propietarias o arrendatarias y se les ha facilitado créditos, asistencia técnica, mercado y precios seguros para sus productos en condiciones de igualdad con los hombres.

- El sostenido crecimiento en el acceso de las mujeres a cargos electivos se observa en diferentes instancias de las distintas asociaciones y el Ministerio de la Agricultura (MINAG).

- El nivel cultural general de las dirigentes campesinas ha aumentado y se ha reportado un incremento 
en el número de mujeres con nivel universitario y nivel medio superior.

- La integración de las mujeres campesinas a la Asociación Nacional de Agricultores Pequeños (ANAP) como socias va en incremento, lo cual implica no sólo la garantía del trabajo, sino los beneficios de la seguridad social, maternidad y vacaciones.

- La Legislación Agraria contempla a la mujer en un plano de igualdad y a su vez, existe una protección específica a mujeres rurales trabajadoras asalariadas en sectores estratégicos en cuanto a la jubilación.

- El aumento de las mujeres beneficiadas con el Decreto Ley 259 y 300, a las que se ha otorgado el usufructo de la tierra, se les confiere además pleno acceso a los créditos y asistencia técnica, entre muchas otras oportunidades. Esta modalidad posibilita a las mujeres aumentar el manejo, control y administración de la tierra y los recursos del sector agropecuario.

ESTUDIOS DE CASOS EN EL SECTOR RURAL Y AGROPRODUCTIVO CIENFUEGUERO. APUNTES METODOLÓGICOS

En la provincia Cienfuegos, la mujer ha escalado a los diferentes niveles de dirección en los sectores 
de la economía. No obstante, a pesar de la consolidación de la presencia de la mujer entre la población activa y el cambio de su papel tradicional en la sociedad, su participación en la esfera de la dirección no se manifiesta por igual en cada una de las actividades empresariales. Tal es el caso del sector agropecuario en Cienfuegos, del cual se presentan 5 estudios de casos en los municipios: Abreus, Rodas, Cumanayagua, Cruces y Lajas, realizados en los años 2012 hasta 2016. En los 5 estudios de casos se presenta como forma productiva principal la asociada a cultivos varios entre los que sobresalen: caña de azúcar, arroz, granos, vegetales, hortalizas y, en el caso de Cumanayagua, el café. Se vinculan a estas formas agroproductivas la tenencia de animales entre ellos: ganado mayor, ganado menor, aves de corral y las meliponas (abejas de la tierra).

Los estudios realizados fueron todos exploratorios y descriptivos, en los que se aplicaron diferentes técnicas de investigación, entre las que se encuentran: entrevistas en profundidad, análisis de documentos y encuestas a familiares y subordinados de las mujeres objeto de estudio. Para esto se planteó como problema de investigación: ¿cómo se manifiestan las relaciones de género en la ocupación de cargos directivos en el sector agropecuario en la provincia Cienfuegos?

Como objetivo general de la investigación se propuso: valorar la situación de la mujer en los cargos de dirección y las relaciones de género que se manifiestan en el sector agropecuario 


\section{6}

LA VENTANA, NÚM. $51 / 2020$

de la provincia Cienfuegos. Los objetivos específicos que se abordaron en los casos de estudios fueron:

- Caracterizar desde la perspectiva de género el sector agropecuario.

- Describir la participación de la mujer en el desempeño de cargos de dirección en el sector agropecuario. - Analizar los factores que condicionan la presencia de mujeres en cargos directivos en el sector agropecuario.

- Identificar las barreras que se presentan en el desarrollo de las mujeres que ocupan cargos de dirección en el sector agropecuario.

La muestra que se utilizó fue intencional: 36 mujeres que se desempeñaron como dirigentes formales en las formas de organización de la producción agropecuaria en Cienfuegos. A todas se les aplicaron entrevistas en profundidad, se realizaron encuestas a los familiares cercanos a estas mujeres dirigentes (86 personas), a subordinados de ambos géneros (121 personas) y a homólogos y superiores (43 personas). 


\section{CARACTERIZACIÓN DE LOS CASOS DE ESTUDIO DESDE LA PERSPECTIVA DE GÉNERO}

En los 5 casos de estudio se evidencia que las eda-

des en las que se encontraban las mujeres dirigentes se muestra en el rango de 25 a 35 años de edad, donde las mujeres se encuentran en una etapa de su vida en las que ya han cumplido alguno de sus proyectos de vida en el plano familiar y el laboral. Dentro de este mismo rango sobresalen las casadas con hijos, mientras que en las edades restantes se encuentran solteras o en unión libre, aunque la presencia de hijos se mantiene.

En los años vinculados al sector agropecuario, el 50\% de estas mujeres no han asumido la superación profesional. Del total de entrevistadas el $25 \%$ son graduadas del nivel superior mientras que el $75 \%$ se encuentra en el nivel medio superior con especialidades y técnicos medios que se relacionan indistintamente con los productos que ofrece el sector agropecuario o la propia producción de las entidades en las que laboran. Se abordaba el tema relacionado con la dirección y si existía una experiencia previa al respecto. El 85\% no posee experiencia previa, pero lo cierto es que éstos son entornos que tradicionalmente han sido ocupados por los representantes masculinos. 
PROCESO DE PROMOCIÓN Y ACCESO

A CARGOS DE DIRECCIÓN EN EL SECTOR RURAL Y AGROPRODUCTIVO CIENFUEGUERO

La promoción y ascenso a los cargos de dirección en la empresa estudiada se relaciona con un proceso mediado por los requisitos y condiciones establecidos nacionalmente, así como las regulaciones ministeriales por las que se rigen las cooperativas seleccionadas. El $83 \%$ de las representantes abogan haber comenzado en los cargos de dirección desde la Dirección de Recursos Humanos, tareas que les fueron conferidas por las habilidades y valores demostrados, pero que, en ocasiones, es necesario resaltar, se encontraban vacantes por diferentes motivos. Pero lo relevante es que una vez asumidas por estas mujeres han tenido un desempeño admirable y reconocido en esta etapa de su trayectoria laboral.

Dentro de las funciones que desempeñan se encuentra la participación activa en los Consejos de Dirección, así como en la toma de decisiones a niveles jerárquicos (entre los que se encuentra aquellos asociados). Ahí son escuchadas sus propuestas, y sus opiniones se tienen en cuenta en la toma de decisiones a nivel empresarial y en aquellas entidades en las que se encuentran ejerciendo la dirección.

Las respuestas de las entrevistadas referidas a la planificación del trabajo fueron dadas en función del sistema de plani- 
ficación que utiliza la organización a mediano y largo plazo, la planificación operativa, y las habilidades que los directivos y directivas entrevistadas utilizan para distribuir sus actividades.

PARTICIPACIÓN DE LA MUJER EN LA

TOMA DE DECISIONES Y LA

ORGANIZACIÓN DEL TRABAJO EN

LOS CARGOS DE DIRECCIÓN EN EL SECTOR RURAL Y AGROPRODUCTIVO

DE CIENFUEGOS

Si bien las mujeres han sido especialmente benefi-

ciarias de políticas que han mejorado su participación en disímiles esferas de la vida, aún existen brechas de género en diversos espacios. Hay que destacar que no resulta fácil comprender la falta de correspondencia entre la participación económica de las mujeres y su protagonismo en la dirección de los procesos productivos, este hecho requiere de un análisis muy complejo acerca de cómo se produce el proceso de incorporación de las mujeres a las empresas y el largo camino que tienen que recorrer para desempeñar la actividad de dirección. Muchas de ellas comenzaron sin ningún tipo de formación profesional y en situaciones muy adversas, tanto desde el punto de vista laboral, como del familiar.

Además, resulta necesario explicar la relación entre el ámbito doméstico y el desempeño de las mujeres en la dirección de las empresas, un elemento que sin dudas tiene una influencia 
decisiva en la proyección social femenina. No puede obviarse que estas mujeres aún cuando tienen un protagonismo social mayor por las responsabilidades que adquieren en la esfera laboral, llevan el peso de las tareas del hogar, incluso cuando el esposo contribuye y otros miembros de la familia desempeñan diferentes funciones. Resulta muy difícil desarrollar una estrategia familiar que no las responsabilice directamente con el funcionamiento del hogar y de la familia. Se refieren a la administración de todos los recursos del hogar, los gastos y las necesidades relacionadas con la familia.

Esto implica que la problemática familiar tenga que definirse hacia patrones más equitativos en el manejo de los asuntos domésticos y las responsabilidades del hogar ante la responsabilidad laboral que tienen. Se sienten útiles en la función que realizan y en el desarrollo de la empresa. Consideran que proyectan desde su espacio la imagen de que la mujer puede desempeñarse en el área que se proponga y para la cual se preparare, no importa lo difícil de la tarea. Para llevar a cabo estas funciones, tienen que realizar mayor sacrificio que el hombre, pues no desatienden las obligaciones del hogar y la familia.

Para conocer cómo planifican el tiempo y las tareas, se les pidió a las entrevistadas que comentaran sobre lo que hacen en un día "normal". Aunque todas mencionan tres momentos importantes en el día (antes de comenzar la jornada laboral, durante, y después), el significado de estos momentos y las actividades que incluyen en ellos son diferentes para mujeres y varones. 
Al responder, las mujeres entrevistadas hacen referencia a un momento que para los hombres es invisible o al menos no hacen mención. Este lapso se sitúa entre la hora de levantarse y la de llegar a la empresa, en el que realizan un grupo de actividades relacionadas con las labores del hogar y el cuidado de los hijos. Por las entrevistas, pareciera que para los representantes masculinos la vida comienza a partir de que llegan a la empresa.

En el caso de las entrevistas que hacen referencia a este tiempo "antes" de llegar a la organización también mencionan que lo dedican a realizar actividades para el cuidado personal y familiar. De forma general, todos los entrevistados utilizan el tiempo "después" de terminada la jornada laboral para realizar actividades de mayor concentración como escribir informes, chequear informaciones económicas, entre otros. Esta forma de trabajar demanda grandes exigencias en términos de tiempo dedicado al trabajo. En el caso de las mujeres es mayor, pues en sus hogares se mantiene la división sexual del trabajo: las labores domésticas y el cuidado de los hijos sigue siendo su responsabilidad.

En el plano del conocimiento, la experiencia de dirección ha significado un acercamiento de las personas, trabajo en equipo y participación en grupos, aprendizaje de gestión, entendimiento y manejo de información, entre otros. También han desarrollado su capacidad de relación con los superiores, subordinados y personas en general que reclaman su atención. 
Han adquirido experiencia organizativa y gerencial, contribuyendo así a los resultados de la gestión empresarial.

Al indagar sobre la forma en que toman las decisiones se exploraron los niveles de intervención en la toma de decisiones: participativa, consultiva o centralizada, así como la orientación de éstas: hacia las personas o hacia las tareas. No se encontraron diferencias significativas sobre este aspecto entre las respuestas de mujeres y varones, quienes privilegian el nivel de consulta y la orientación a las tareas en la toma de decisiones, basados en hechos "sólidos, objetivos, racionales".

Se aplicaron encuestas a subordinados femeninos y masculinos de las mujeres dirigentes, éstos poseen una edad promedio de 44 años. El $60 \%$ son técnicos medios, el 30\% graduados universitarios y el $10 \%$ son bachilleres. Dentro del cuestionario se les pedía a los subordinados que caracterizaran a su jefa; el 30\% manifiesta que se preocupa por sus subordinados, por las tareas que estos deben desempeñar, pero que al mismo tiempo muestran interés no sólo por su vida laboral sino también por la familiar, diferencia notable con respecto a los representantes masculinos que actualmente se desempeñan como dirigentes en dicha empresa.

El $60 \%$ afirma que sus criterios son escuchados por sus superiores, en este caso las mujeres dirigentes transmiten y tramitan las preocupaciones y sugerencias a los integrantes a las escalas jerárquicas superiores. En el caso de la calidad de la información que reciben por parte de las mujeres dirigentes 
que son las que los representan ante el Consejo de Dirección, el $60 \%$ plantea que la calidad y las informaciones recibidas son las adecuadas, transmitidas de forma cordial, cortés y en las ocasiones en las que es necesario realizar llamados de atenciones a algunos de los subordinados, dicha conversación se realiza a puertas cerradas, mientras que los estímulos son formalizados ante la totalidad del colectivo laboral.

\section{FACTORES QUE CONDICIONAN LA PRESENCIA DE MUJERES EN CARGOS DIRECTIVOS Y LAS BARRERAS QUE FRENAN SU DESARROLLO EN EL SECTOR RURAL Y AGROPRODUCTIVO CIENFUEGUERO}

La limitación fundamental que señalan las directivas se relaciona con la falta de motivación hacia la mujer. Plantean que la fuerza laboral femenina existente en el sector agroproductivo y rural está preparada, con condiciones y experiencia para dirigir, sin embargo, a pesar de existir mujeres incorporadas a la reserva de cuadro, la mayoría de las féminas no poseen disposición de asumir puestos de dirección ya que no se encuentran motivadas. Coinciden en señalar que la desmotivación está influenciada por las malas condiciones laborales, los horarios ocupados fuera de la jornada laboral habitual y la remuneración que perciben los cuadros, que no se 
encuentra en correspondencia con los niveles de responsabilidad, sacrificio, abnegación y entrega.

No obstante, las entrevistadas definen el desempeño de su puesto como una satisfacción y un desafío, ya que, a pesar de considerar al sector de la agricultura con pocos atractivos, les gusta la función que desempeñan. Mencionan algunas dificultades en el ejercicio de la dirección como: tener que adaptarse a las estrategias de dirección de la empresa, que en algunos casos no comparten; carencia de recursos y dificultades con las condiciones laborales; falta de preparación y superación; carencia de reconocimiento y estimulación a la labor de dirección.

Existe un elevado nivel de interrelación entre las diferentes barreras, se muestra que el factor fundamental que limita la participación de la mujer en el acceso a cargos directivos en el sector rural y en especial en el ámbito agroproductivo. La falta de una infraestructura social es otro elemento que influye de manera significativa en el desarrollo profesional de las mujeres. Las carencias originadas por la situación socio-económica que atraviesa el país, inciden en la disposición del tiempo de las féminas, a partir de la escasez de instalaciones de servicios, tales como lavanderías, círculos infantiles, hogares de ancianos y otros, lo que refuerza el papel tradicional de la mujer y la limita considerablemente a participar en igualdad de condiciones que los hombres en el desarrollo en la esfera de la dirección.

En sentido general, la desigual distribución de responsabilidades familiares, los factores asociados a la función reproductiva, 
la multiplicidad de roles femeninos, la falta de servicios de apoyo doméstico, así como no tener en cuenta la situación de la mujer al evaluar su desempeño, se traduce en un desdoblamiento del tiempo y las energías de las mujeres, lo que incide negativamente en sus oportunidades profesionales y laborales que deben sortear ante el ejercicio de la dirección.

\section{CONCLUSIONES}

- La situación de la mujer cooperativista no difiere mucho de la situación de la mujer rural en general. Las mujeres miembros de las UBPC, CPA y CCS, propietarias o usufructuarias, con escasas excepciones, viven en ambientes donde predominan prejuicios sobre las mujeres y los hombres. Las mujeres rurales están en desventaja económica y social respecto a otras mujeres y hombres cubanos.

- Existe un reconocimiento del trabajo desempeñado por las mujeres en la labor de dirección por parte de sus homólogos masculinos y de sus subordinados, pues son varios los ejemplos donde se obtuvieron excelentes resultados producto del empuje, consagración, organización y capacidad de motivación de ellas que estaban al frente de la actividad.

- Las barreras que condicionan la presencia de las féminas en cargos de dirección en el sector agropecuario 
cienfueguero se asocian con: la falta de apoyo en la superación profesional de las mujeres, dificultades para priorizar entre profesión y familia, el peso de los estereotipos sociales que definen roles y tareas de acuerdo al género. Las labores de la casa recaen sobre la mujer, así como la responsabilidad con los hijos y las personas mayores, la presión familiar, el estrés y otras son elementos que desmotivan a las mujeres para desempeñarse ante los cargos directivos.

\section{Bibliografía}

Álvarez, M. (1998). Mujer y Poder en Cuba. Temas (14), 13-25.

BAUTE, M. (2010). La mujer en la Educación Superior, su participación en la actividad docente investigativa y de gestión en la Universidad de Cienfuegos (Tesis Doctoral). Universidad de Cienfuegos, Cienfuegos.

DíAZ, I. y CALDERón, L. (noviembre de 1997). Liderazgo femenino en las organizaciones. Ponencia presentada en el evento Mujeres en los umbrales del siglo XXI. La Habana.

Fuller, N. (2006). Los estudios de género en el ámbito sudamericano. Global Education Digest. Montreal: UNESCO. Recuperado de http:/ /www.inicia.es/de/cgarciam/Fuller.html

González, G. (2005). El empoderamiento femenino en las empresas industriales. En C. Proveyer (Coord.), Selección de lecturas de Sociología y Política social de Género (pp. 107-111). La Habana: Editorial Félix Varela. 


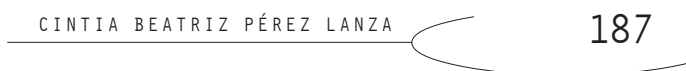

Hernández, A. y Arteaga, C. (2013). Gestión integral cooperativa. Guía para formadores y facilitadores. La Habana: MINAG.

JACOBS, J. A. (1992). Womens entry into management: trends in earnings, authority and values among salaried managers. Administrative Science Quarterly, 37(2), 282-301.

KESNER, I. (1989). Director's characteristics and committee membership an investigation of type, occupation, ternure and gender. Academy of Management Journal, (31), 66-84.

NuÑEZ, M. (2001). Los estudios de género en Cuba y sus aproximaciones metodológicas, multidisciplinarias y transculturales (1974-2001). La Habana: Centro de Estudios de Migraciones Internacionales.

SCOTT, J. (1996). El género: Una categoría útil para el análisis histórico. En M. Lamas (Comp.), El género: la construcción cultural de la diferencia sexual (pp. 265-302). México: PUEG-UNAM.

SMIRCICH, L. Y CALÁs, M. (1983). Concepts of Culture and Organizational Analysis. Administrative Science Quarterly, 28(3), 339-358.

SNAVELY, B. K. (1993). Managing conflict over the perceived progress of working women. Business Horizons, 36(2), 17-22.

TARRÉs, M. L. (Comp.). (1992). La voluntad de ser. Mujeres en los noventa. México: El Colegio de México.

TuÑón, J, y GonZÁLEZ, S. (1997). Familias y mujeres en México: del modelo a la diversidad. México: El Colegio de México, Programa Interdisciplinario de Estudios de la Mujer.

Vasallo, N. (2004). Mujeres Cubanas. Historia, contradicciones y cambios contemporáneos. Ottawa, Canadá: Carleton University. 